\title{
AC-Pulse Modulated Electrohydrodynamic Jet Printing and Electroless Copper Deposition for Conductive Microscale Patterning on Flexible Insulating Substrates
}

Hantang Qin, Jingyan Dong, and Yuan-Shin Lee*

Edward P. Fitts Department of Industrial and Systems Engineering, North Carolina State University, Raleigh, NC 27695, U.S.A.

*: Correspondent Author’s E-mail: yslee@ncsu.edu 


\begin{abstract}
This paper presents a novel micro-manufacturing method for fabrication of electrical features and patterns on highly insulating substrates and flexible substrates based on high-resolution AC-pulse modulated electrohydrodynamic jet (e-jet) printing of silver nanoink as seed layer followed by electroless copper deposition. Traditional ink jet printing method is limited in printing resolution which is determined by dimension of printing nozzle and dimension of droplets. Traditional e-jet printing has the disadvantage of residual charge problem especially for highly insulating substrates which cannot dredge remained charge of printed droplets, resulting in distorted electrostatic field and low printing controllability. Meanwhile, for printing of liquid phase ink, feature resolution contradicts with the required thickness, which is a key factor of conductivity of printed patterns. In this paper, a novel ACmodulated e-jet printing technique is applied to neutralize charges on substrates by switching polarity of consequent droplets for direct printing of high-resolution conductive silver patterns on insulating substrates. Electroless copper deposition is introduced in the fabrication process to solve the thickness problem of the resulting features. Variables of fabrication process, including amplitude and frequency of AC-pulsed voltage, plotting speed, curing temperature, number of layers, concentration of solution for copper growth, were identified to achieve reliable and conductive printed patterns. Sub-20 $\mu$ m silver tracks with resistivity about 3.16 times of bulk silver were successfully fabricated. We demonstrated that ac-pulse modulated e-jet printing followed by electroless copper deposition can produce high resolution conductive patterns with improved thickness on insulating substrates and flexible substrates, which can be applied to direct printing and micro scale patterning for flexible electronics and wearable devices applications.
\end{abstract}

\title{
Keywords
}

Electrohydrodynamic jet printing; electroless copper deposition; AC-pulse modulation; direct printing; micro-manufacturing. 


\section{Introduction}

Direct writing techniques such as additive printing show advantages over traditional copper foil etching process for RFID and other flexible electronic applications because of its simplified procedures, compatibility to continuous process, printability for all kinds of materials, and lower cost [1]. Conventional ink jet printing approaches are based on thermal or acoustic formation and ejection of liquid droplets through nozzles [2]. The fabrication processes are successfully applied in electronics [3-6], drug delivery systems [6], micromechanical devices [7, 8] and other areas. The maximum resolution using thermal or acoustic theory is $20-30 \mu \mathrm{m}$, resulted from the fact that the diameters of the droplets are typically bigger than $20 \mu \mathrm{m}$ [9]. Even though with assistant technique, e.g., by combing lithography into these ink jet printing to confine the liquid flow [10], it remains a challenge to for electronics research and industry community to achieve the sub-micro level in fabrication processes.

Researchers began to introduce electrohydrodynamic theory into additive inkjet printing. The electrohydrodynamic mechanism was first proposed by Zeleny back in 1917 [11]. The multiplicity of electrohydrodynamic spraying modes has been studied ever since and different functioning modes such as cone-jet, multijet, microdripping, simple jet and ramified jet was reported [12]. Electrohydrodynamic jet (e-jet) printing is a pattern method using electric fields to generate fluid flows to deliver inks to a substrate working in cone-jet mode. It offers advantages compared with traditional thermal or acoustic based inkjet printing technologies. The resolution of printed patterns can be dramatically improved by applying electrical fields. When the liquid ink is supplied to an electrical potential high sufficiently, a stable cone will be formed at the tip of nozzle, emitting a stable jet on its summit. Lee et al. reported printed patterns with resolution down to $40 \mathrm{~nm}-1.8 \mu \mathrm{m}$ using electrohydrodynamic printing to optimize physical properties of the liquid droplet by controlling parameters [13]. Silver nano ink has been reported to be used as electric circuits $[14,15]$. At the same time, silver can also be used as catalyst for copper electroless deposition.

However, before e-jet printing can be adapted to manufacturing printing processes, there are still plenty of obstacles remain to be overcome. First, e-jet printing is a patterning method that uses a fine jet generated at the apex of ink cone of an electrospray in cone-jet mode [16], which are determined by experimental parameter, such as electric field, conductivity and viscosity of fluids and flow rate [17]. Numerical analysis of formulations and relationships are hard to develop among many parameters. Lee et al. (2012) have tried to propose a formulation between droplet size, surface tension and applied voltage, but it was not suitable for jet formation situations [18]. More research about conditions and parameters for e-jet printing needs to be conducted [43]. Second, fabrication of thick features in micro and nano scale is attractive for applications of e-jet printing in circuit fabrication towards high conductivity. However, highly conductive silver tracks with such high resolution are tremendously difficult to print. Since single printed layer would be only $50 \mathrm{~nm}$ thick, it is necessary to print multi-layers for better conductivity. However, multi-layers would play a negative role on resolution. Smaller diameter of nozzle is another key factor for high resolution, while blockage of the nozzle is unavoidable and conductivity of the silver line would be even poor with thinner thickness[19]. Besides, it still remains to be challenging to print conductive silver tracks on highly insulating materials such as Ajinomoto build-up (ABF) films, and flexible substrates such as polyethylene terephthalate (PET) films.

Copper has been widely used for interconnects in integrated circuits and electronic packages because of its low resistivity and high electromigration resistance. Electroless deposition of metals such as copper, silver, gold and nickel have been developed since 1990s and been widely used for production of fine metal patterns in printed circuits [20]. The process occurs by a redox process in which the cation of metal to be deposited is reduced by a reducing agent at the surface of catalysts or induced areas used to initiate the deposition. The redox process generally takes place only on catalyzed area, such as activated surface or metal seeds like silver or palladium, which function as catalyst [21]. Selective deposition can be achieved by producing patterned catalysts. Hidber et al. reported patterning surfaces with palladium catalyst by microcontact printing of colloids, which enables selective copper metallization by electroless 
deposition on areas of surface activated by deposited colloids. They generated metallic features with submicron dimensions and film thicknesses greater than $1 \mu \mathrm{m}[22]$.

Generally, electroless metallization is cost-effective compared with sputtering, chemical vapor deposition, and evaporation, since it does not require vacuum equipment or high power supplies. The elimination of expensive palladium or expensive metal catalyst would reduce the cost of electroless plating. Silver was selected in our research due to its catalytic properties, cost and compatibility to our ejet printing system for thickness increase. However, whether copper can be selectively deposited on silver nano ink still remains unknown and characterization of the process need to be studied.

In this paper, we proposed a combined fabrication technique by printing silver patterns on the substrate followed by electroless copper deposition on printed silver seeds. The process is capable of fabricate sub-20 $\mu \mathrm{m}$ patterns with increased thickness up to $15 \mu \mathrm{m}$. The use of e-jet printing of highly conductive silver tracks based on AC-pulse modulated voltage with sub-20 $\mu \mathrm{m}$ resolution was presented in the paper. High resolution printed metallic silver tracks with resistivity about 3.16 times of bulk silver showed great potential applications in printed electronics. The fabrication process proposed in the paper is able to on demand print metal tracks on highly insulating materials. The method of depositing copper based on printed silver catalyst is capable of growing copper with thickness up to $15 \mu \mathrm{m}$ and resistivity about four times of bulk copper, which provide a good replacement of traditional palladium catalyst based deposition in terms of cost of process. The combined fabrication technique can be further adapted in integrated circuits, packaging substrates, interconnectors, transistors, and printed circuit boards for flexible electronic devices or prototyping. Our process demonstrates a new strategy, combination of direct e-jet printing of silver seeds and electroless metal deposition, for fabrication of thick metal features with high resolution. Details of the proposed new method are presented in the following sections.

\section{Methods and Materials}

\subsection{AC-pulse modulated e-jet printing system and process}

As shown in Fig. 1, the e-jet printing system proposed in this research consists of a three-axis (XYZ) stage, a dispensing system with pressure regulator, a nozzle, substrates, and a high AC voltage power supply. The required flow rate of e-jet printing is provided through pneumatic syringe with a 5 psi maximum pressure and 0.05 psi resolution. The nozzle is functioning as electrode, and used to produce a jet containing organic silver nanoparticles, which are supplied by the syringe. Desired voltage is generated, amplified and applied between nozzle and electrode. Waveform of the AC-pulsed voltage is fixed while amplitude and frequency can be adjusted. The ac-pulsed voltage is programmed using a function waveform generator (Agilent 3320A), and amplified by a high voltage amplifier (Trek 610A) for the process.

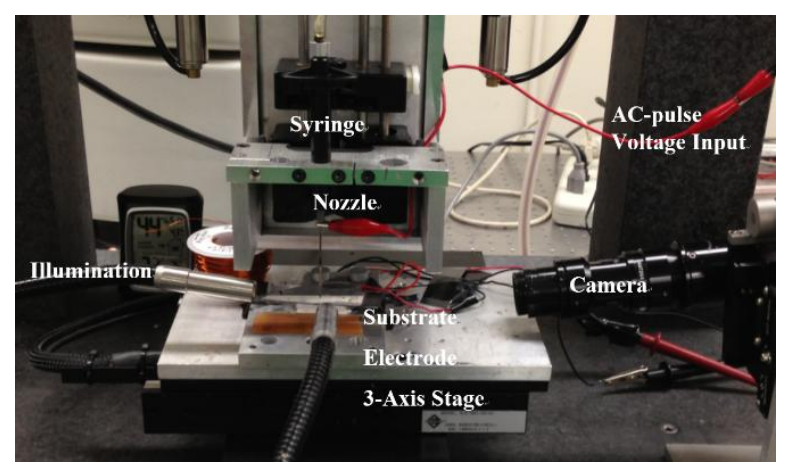

Fig. 1: Setup of E-jet printing system: AC-pulse modulated voltage is applied between nozzle and bottom electrode under substrate 
The stage could provide a displacement for substrate to control trajectory of tracks by move 3-Axis stage in X-Y directions. The motion range of the stage is $100 \mathrm{~mm} \times 100 \mathrm{~mm} \times 50 \mathrm{~mm}$ with $100 \mathrm{~nm}$ repeatability. This fabrication process is capable of on demand printing multi-layers on substrate by programming the movement of 3-axis stage. A video camera is used to real-time monitor the printing process.

In the last few years, several research works were presented on patterning of metal nanoparticles through e-jet printing on glass and silicon substrate [23-27] and droplet analysis using different forms of voltage [28-31]. However, there were always controllability and residue charge problems. In the study, AC-pulse modulated voltage [32-33] was adapted to improve controllability and reduce residue charge accumulation for flexible electronic applications where substrates would most likely be highly insulating.

(a)

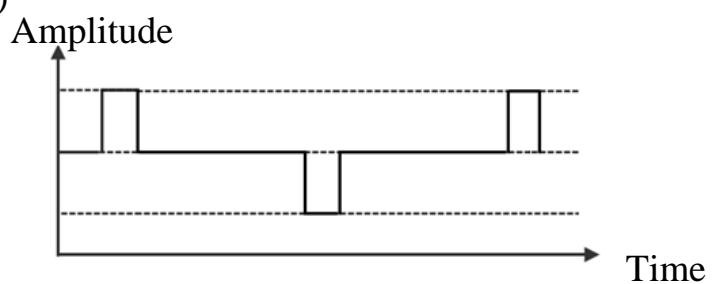

(c)

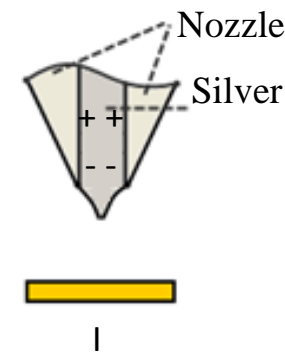

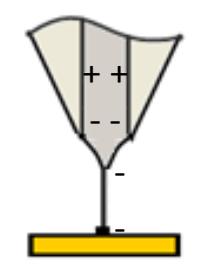

II

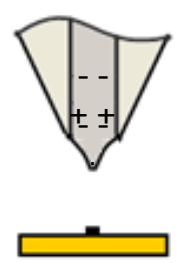

III

(b)

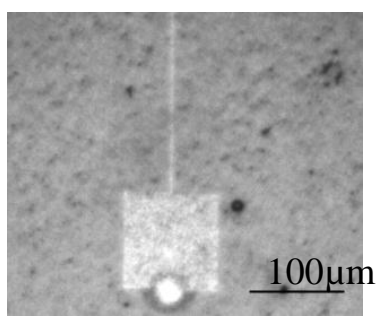

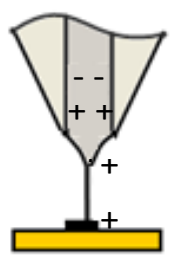

IV

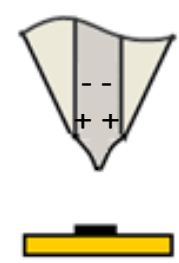

V

Fig. 2: (a) AC voltage signal with duty ratio of one, (b) 30-layer straight silver track with line width of $2.3 \mu \mathrm{m}$ printed on $\mathrm{ABF}$, (c) mechanism of e-jet printing using AC voltage printing on highly insulating substrate

Printing on insulating substrate using DC voltage is very difficult. Since the substrate will never transmit or neutralize any charges of droplets and inks. The charged ink accumulates on the substrate and then affected the whole electric field between nozzle and substrate. Two scenarios will happen: (1) the jet is affected by net charge of previously printed ink, resulting in deflection in printing direction; (2) the jet is completely stopped, resulting in discontinuous tracks. AC-pulse modulated voltage input can solve this problem. The mechanism of fabrication process of e-jet printing using AC-pulsed voltage is shown in Fig. 2. Due to the AC pulse signals, positive and negative charges are induced on the meniscus of the ink at the tip of the nozzle sequentially, forming positive and negative jet sequentially. Fig. 2 (a) shows a circle of AC signal. In Fig. 2 (c)-(II), negative charged jet would print negative ink on Ajinomoto build-up films (ABF) substrate first, resulting in negative ink printed on ABF in Fig. 2 (c)-(III). As electrode polarity changes, a positive charged jet is generated (Fig. 2 (c)-(IV)) and printed along the tracks. Since the duty ratio of the signal is one, the negative printed tracks would be neutralized by these positive charges. The printed tracks return to an electrically neural state that is ready for another period of ejections. An electronic pattern with a $100 \mu \mathrm{m}$ width pad and a $2.3 \mu \mathrm{m}$ silver track was obtained on ABF film using AC-pulse modulated voltage with nozzle outer diameter of $7 \mu \mathrm{m}$ and inner diameter of $5 \mu \mathrm{m}$, as shown in Fig. 2 (b). To acquire highly conductive micro tracks, multilayered printing was attempted by direct placing the deposition on top of each other in the study. 


\subsection{Electroless copper deposition on printed silver seeds}

Fig. 3 presents a schematic outline of the combined fabrication process with e-jet printing and electroless deposition. The whole process can be adapted into traditional continuous fabrication process or roll-to-roll manufacturing. We used e-jet printing to deposit silver seeds patterns on the substrate. The substrate was then immersed in the plating solution. The electroless copper deposition occurred only where silver seeds were printed. A challenge for e-jet printing at this moment is improving resolution of printed patterns while maintaining high conductivity at the same time. In our previous research [32-35], we reported printing of silver filaments with line width down to $2 \mu \mathrm{m}$ and good conductivity. However, the thickness of printed line is about $300 \mathrm{~nm}$ even though with multi-layer printing due to low viscosity of silver ink (tendency to split out instead of accumulate layer by layer). That's the motivation for introducing electroless copper deposition into the procedures to increase the thickness of patterns.

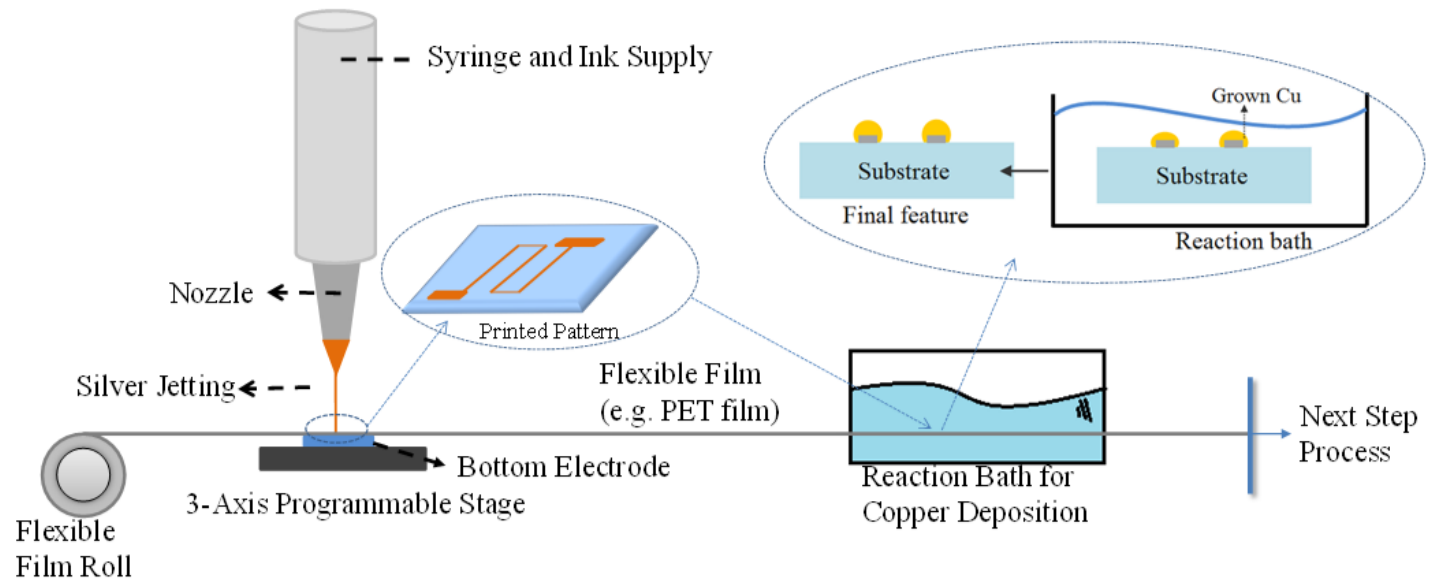

Fig. 3: Schematic outline of procedure for e-jet printing of silver seeds and subsequent copper deposition. Metallization occurred only where substrate was activated with silver seeds

Electroless deposition of metal is based on reaction between copper ions and a chemical reducing agent in the electrolyte. The process is initiated by a catalyst on the substrate surface. Once the target metal is precipitated out at the first place, the process transforms into autocatalytic process. The precipitation will keep going on where the precipitated metals oxidize reducing agent [36]. The substrates or catalysts will no longer influence electroless copper plating after several atomic layers of copper deposition. The schematic of electroless copper deposition with e-jet printed silver seeds is shown in Fig. 3.

The mechanism of electroless copper deposition has been thoroughly studied [37]. There are plenty of empirical laws proposed to measure the reaction orders [38]. Traditional electroless plating baths are typically composed of a metal salt such as metal ion sources like $\mathrm{CuSO}_{4}$ and $\mathrm{AgNO}_{3}$, chelating agent to prevent formation of undesired compounds, and a reducing agent (formaldehyde). The reactions are typically catalyzed only on noble metals, including $\mathrm{Pt}, \mathrm{Pd}, \mathrm{Ag}, \mathrm{Au}, \mathrm{Rh}$, or Ir [39]. Overall, electroless copper deposition can be generalized into the anodic and cathodic reactions [40]:

Anodic half reaction: $\quad 2 \mathrm{HCHO}^{2}+4 \mathrm{OH}^{-} \rightarrow 2 \mathrm{HCOO}^{-}+2 \mathrm{H}_{2} \mathrm{O}+\mathrm{H}_{2} \uparrow+2 \mathrm{e}^{-}$

Cathodic half reaction: $\quad \mathrm{CuL}_{\mathrm{n}}^{2-\mathrm{n}^{*} \mathrm{~m}} \leftrightarrow \mathrm{Cu}^{2+}+\mathrm{nL}^{-\mathrm{m}}, \mathrm{Cu}^{2+}+2 \mathrm{e}^{-} \rightarrow \mathrm{Cu}$

where L represents complexing agents, such as the most common complexing agents used for formaldehyde-based electroless copper deposition is ethylenediaminetetraacetic acid (EDTA) and triethanolamine (TEA), and $\mathrm{m}$ represents the charge of chelating agent. 


\subsection{Materials and characterization}

\subsubsection{Materials}

Silver nanoparticles (Silverjet DGP-40LT-15C) were purchased from Advanced Nano Products Co., Ltd. with 20-35 wt.\% silver nanoparticles and about 65-80\% triethylene glycol in weight with small amounts of surfactants to prevent agglomeration between silver nanoparticles. Nano silver particles are uniformly dispersed in the solvent as high solid content with low viscosity for ink jet applications. The geometric diameter of the silver nanoparticles is below $50 \mathrm{~nm}$. Density of the dispersion nanoparticles is $1.45 \mathrm{~g} / \mathrm{mL}+/-0.05 \mathrm{~g} / \mathrm{mL}$ at $25^{\circ} \mathrm{C}$. The viscosity is $10-18 \mathrm{cP}$ and surface tension is $35-38 \mathrm{dyn} / \mathrm{cm}$. General curing temperature recommended by the company is $120-200{ }^{\circ} \mathrm{C}$. In our experiments, the printed substrates were heated to $180{ }^{\circ} \mathrm{C}$ for 20 minutes to remove solvent before plating reactions.

Three types of substrates used in the study are microscopic glass slides $(75 \mathrm{~mm} \times 25 \mathrm{~mm} \times 1 \mathrm{~mm})$, and two highly insulating substrates, $1 \mathrm{~mm}$ thick insulating ABF coated on glass slides, and $1 \mathrm{~mm}$ thick flexible PET film, which are widely used for high density circuits, electronic packaging and flexible electronics. A dual complexing agent system using EDTA and TEA was employed in our copper deposition process. We used the following copper plating bath: Solution A contains $\mathrm{CuSO}_{4}$ (Alpha Chemical Inc.) as metallic source, EDTA and TEA (Sigma-Aldrich Inc.) as complexing agents, $\mathrm{NaOH}$ to adjust $\mathrm{pH}$, and distilled water. Solution $\mathrm{B}$ is an aqueous formaldehyde solution $(36.5 \% \mathrm{wt} \%$, Thermo Fisher Scientific Inc.). The two solutions A and B were mixed before the bat was used.

\subsubsection{Experimental procedures}

In this paper, the experiments were conducted and details are presented as follows:

(1). For the pretreatment of substrates in order to calibrate, glass substrates were cleaned in an ultrasonic bath for 10 minutes in water, 10 minutes in methanol and 10 minutes in acetone, and 10 minutes in de-ionized water to remove any organic and inorganic contaminants from the substrate.

(2). For AC-pulse modulated e-jet printing, first, a jet was obtained by applying high amplitude ACpulsed voltage after silver particles was supplied to the nozzle through the syringe. The tracks were formed by continuously moving the substrate controlled by 3-Axis stage. The AC-pulsed voltage with different amplitudes and frequencies were provided by the signal generator. Multi layered printing was attempted by direct placing the deposition on top of each other. After the tracks formed, curing process was conducted by heating at ramped temperature with atmospheric pressure on a heating plate. During the printing experiments, a short nozzle-substrate distance of $20 \mu \mathrm{m}$ was chosen. The effects of process parameters such as number of layers, curing temperature on resistivity of silver tracks were investigated to obtain the optimal conditions for printing high-conductive silver tracks.

(3). For electroless copper deposition, we employed concentrations of $12 \mathrm{~g} / \mathrm{L}$ EDTA and $24 \mathrm{~mL} / \mathrm{L}$ TEA in our bath in order to get a stable solution with both relative higher deposition rate and more stable solution reaction intensity from our previous research [41]. We used CuSO4 concentration 9g/L, making sure copper particles inside the solution are enough to grow on silver seeds. The substrates will be immersed into solution at temperature $35^{\circ} \mathrm{C}$ for 30 minutes, extracted, rinsed in deionized water and dried at room temperature. All the samples were then examined by optical microscope and profilometer to find out the thickness increase.

The morphology of the printed tracks was investigated by an atomic force microscope (AFM) (Park Systems, XE-70). The electrical resistance was characterized by a two point probe measurement method. The grown copper is observed by Veeco Dektak 150 Profilometer for topography and copper grown.

\section{Results and Discussion}




\subsection{Characterization of AC-pulse modulated e-jet printing}

In this paper, required voltage amplitude and frequency for e-jet printing were experimentally characterized for stable working conditions. Details of the characterization of the AC-pulse modulated ejet printing are presented in the following sections.

\subsubsection{Effects of AC-pulse voltage}

In AC-pulse modulated e-jet printing, when charge accumulates on the ink meniscus at the nozzle, a fine jet down to nano scale can be generated. The electric stress resulting from applied voltage deforms the meniscus into cone jet mode, a functioning mode of electrohydrodynamic theory. It is critical to find proper voltage amplitude and frequency of the pulsed signal for process development. In the study, required voltage amplitude and frequency for e-jet printing were experimentally characterized for stable working conditions.

(a)

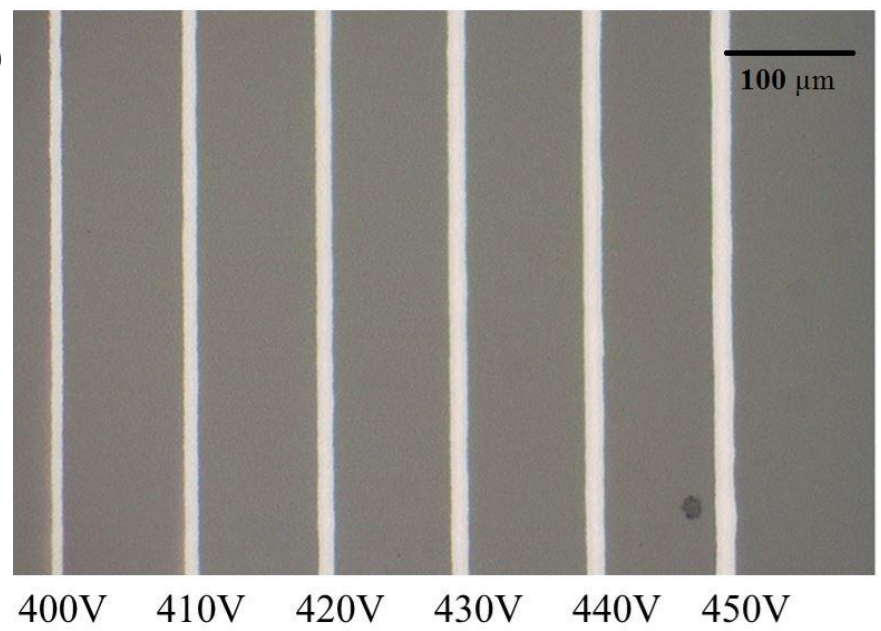

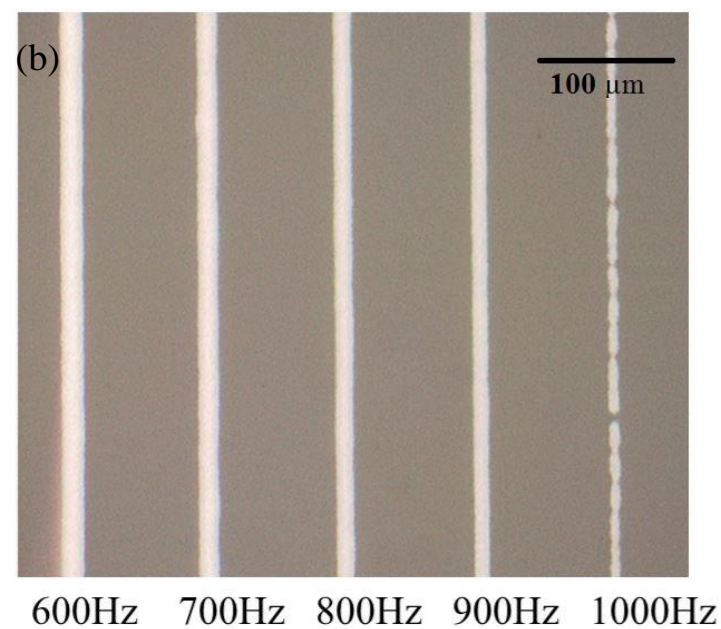

Fig. 4: Printed droplets: (a) at different amplitudes of AC voltage, and (b) at different pulse frequencies

To find the effect of amplitude of voltage pulse on e-jet printing, we printed silver tracks with different voltage while choosing a fixed pulse frequency of $1000 \mathrm{~Hz}$ and duty rate of $10 \%$. The diameter of nozzle at the tip was $2 \mu \mathrm{m}$ and feed rate was $9 \mathrm{~mm} / \mathrm{s}$. As shown in Fig. 4(a), it can be clearly observed that with increasing amplitude, the printed line width of silver tracks was increased. The effect of the voltage is similar to printing results based on DC voltage, because larger amplitudes would result in a greater charge accumulation at the meniscus of nozzle tip and thus bigger droplets sizes.

To study the effect of pulse frequency, the first thing would be figure out the relationship between pulse frequency and printing frequency. When the speed of stage is known, the printing frequency can be measured by printing dots on the substrates. It is verified that printing frequency is exactly twice as pulse frequency, indicating that a pulse, either positive or negative, will result in the formation of a droplet. This is also the reason why charges on the substrate can be neutralized for continuous patterning where two consequent droplets show opposite polarity. We used a fixed voltage of $400 \mathrm{~V}$, a fixed duty rate of $10 \%$, and plotting speed of $9 \mathrm{~mm} / \mathrm{s}$ to print silver tracks on glass substrate with different frequencies. As shown in Fig. 4(b), a reduced line width of printed silver tracks was observed with increasing frequency. With the same duty cycle and amplitude of voltage, larger frequency will result in shorter duration for charge accumulation and thus a smaller droplet. We can notice a little bit discontinuous pattern when frequency was $1000 \mathrm{~Hz}$, at which duration time was too short for charge accumulation. 
It was noticed that, at the critical threshold, even some small variation may result in a difference between continuous and discontinuous patterns. For example, the experiments presented in Figures 4(a) and 4(b) were carried out with the same process parameters by using two sets of prepared nozzle needles. It is noticed that the continuity patterns of the leftmost printed line in Fig. 4(a) and the rightmost printed line in Fig. 4(b), with the same parameters, are slightly different because two similar nozzles (but prepared separately) were used during the experiments. It is noticed that, at the continuity critical threshold, even a tiny variation of nozzle tip may result in slightly different frequency threshold for continuous features. An optimal balance between amplitude and frequency will generate the smallest droplets with continuous features when charge accumulation is enough for continuity.

\subsubsection{Effects of plotting speed and calculation of droplet sizes}

We investigated the effect of plotting speed on printing process and silver tracks while the jetting speed and ink flow were kept constant by using fixed voltage. A $7 \mu \mathrm{m}$ Nozzle was chosen with a fixed $\mathrm{AC}$ voltage of $400 \mathrm{~V}, 1000 \mathrm{~Hz}$ and duty rate of $10 \%$. The distance between the nozzle and substrate was 20 $\mu \mathrm{m}$. Based on the measurements of printed silver droplets, we were capable of calculating and analyzing the mechanism of printing process. We assumed the falling droplets are perfect sphere and printed dots are cylinder shape with average height and diameter measured from AFM image. Another assumption is that the triethylene glycol in the droplets would be volatilized during and soon after falling.

(a)

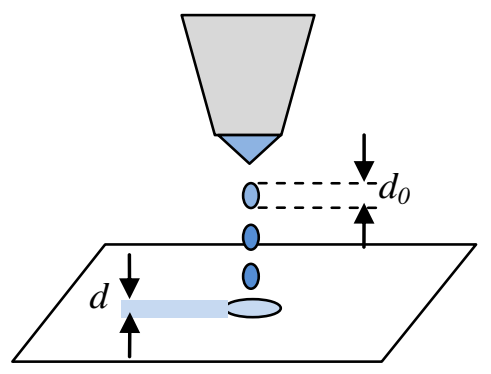

(b)

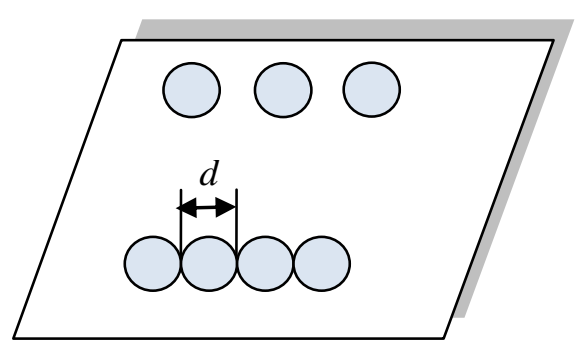

$8.5 \quad 7.5$

\section{5}

12.5
11.5
$\mathrm{~mm} / \mathrm{s}$
10.5
$\mathrm{~mm} / \mathrm{s}$ $\mathrm{mm} / \mathrm{s} \quad \mathrm{mm} / \mathrm{s}$
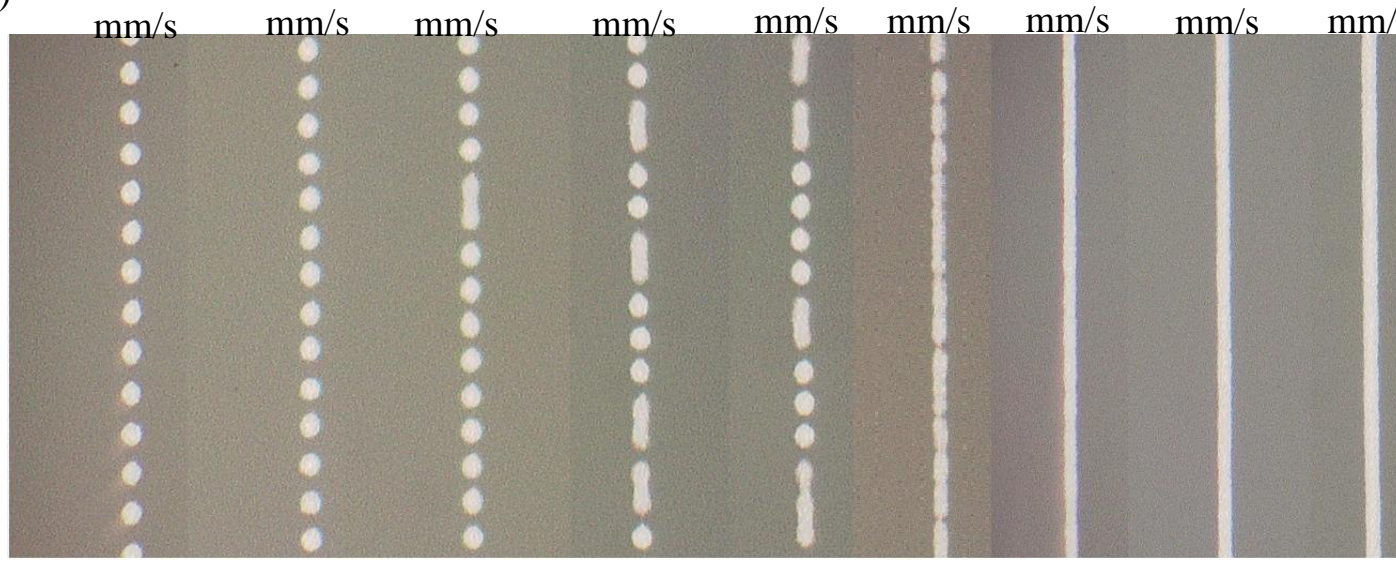

$\underline{20 \mu \mathrm{m}}$

Fig. 5: Sketches on a microscopic of (a) calculation of diameter of falling droplets; (b) requirements to obtain connective silver tracks; (c) Effect of plotting speeds: plotting speed increased from $7.5 \mathrm{~mm} / \mathrm{s}$ to $16.5 \mathrm{~mm} / \mathrm{s}$ with reduced line width and discontinuous pattern

As shown in Fig. 5 (b), in order to print connective silver tracks instead of separate dots on substrates, two adjacent droplets has to be at least tangent to each other. The time between two adjacent 
droplets is half of cycle time of AC voltage. The maximum plotting speed can be calculated using the following Eq. (1):

$$
v_{\max }=\frac{d}{T_{d}}=\frac{2 d}{T}=2 d f, \text { and } T=\frac{1}{f}, T_{d}=\frac{T}{2}
$$

where $T=$ cycle time of AC voltage, $f=$ frequency of AC voltage, $T_{d}=$ time between two adjacent droplets, $d=$ diameter of printed droplets, and $v_{\max }=$ plotting speed.

As shown in Fig. 5 (a), once a droplet is formed, it will fall off and flatten on the substrate to form a dot. The volume of droplets and dots are equal, as shown in Eq. (2).

$$
V_{0}=V_{1}
$$

where $V_{0}=$ actual volume of falling droplets, and $V_{l}=$ volume of printed droplets. We can also calculate the volume of falling droplets and volume of printed droplets using Eq. (3).

$$
\begin{aligned}
& V_{0}=\frac{4}{3} \times \pi \times\left(\frac{d_{0}}{2}\right)^{3}, V_{1}=\pi \times\left(\frac{d}{2}\right)^{2} \times h \\
& V_{1}=V_{0}, \rightarrow \pi \times\left(\frac{d}{2}\right)^{2} \times h=\frac{4}{3} \times \pi \times\left(\frac{d_{0}}{2}\right)^{3} \\
& d_{0}=2 \times \sqrt[3]{\frac{3 V_{0}}{4 \pi}}=\sqrt[3]{\frac{3 \times d^{2} \times h}{4}}
\end{aligned}
$$

where $d_{0}=$ actual diameter of falling droplets, $d=$ line width of printed tracks (diameter of printed droplets), and $h=$ average height of printed droplets.

Once we measure line width of printed tracks and average height of printed droplets, the actual size of falling droplet can be calculated using Eq. (5). The cross section of printed silver dots has an average height of $30.73 \mathrm{~nm}$ and an average line width of $6.06 \mu \mathrm{m}$. The maximum speed for connective silver tracks was $12.12 \mathrm{~mm} / \mathrm{s}$ and the actual diameter of falling droplets was $0.92 \mu \mathrm{m}$. Using Eq. (1) and Eq. (5), one can calculate the plotting speed $v_{\max }$ and the actual diameter of falling droplets $d_{0}$ shown as follows:

$$
\begin{aligned}
& f=1000 \mathrm{~Hz}, d=6.06 \mu \mathrm{m}, h=30.73 \mathrm{~nm} \\
& T=\frac{1}{f}, T_{d}=\frac{T}{2}, v_{\max }=\frac{d}{T_{d}}=\frac{2 d}{T}=2 d f=12.12 \mathrm{~mm} / \mathrm{s} \\
& d_{0}=2 \times \sqrt[3]{\frac{3 V_{0}}{4 \pi}}=\sqrt[3]{\frac{3 \times d^{2} \times h}{4}}=0.92 \mu \mathrm{m}
\end{aligned}
$$

When plotting at a small plotting speed, we were able to acquire stable silver tracks. As the plotting speed increased, the width of silver tracks reduced gradually, as shown in Fig. 5 (c) with plotting speed increased from $7.5 \mathrm{~mm} / \mathrm{s}$ to $10.5 \mathrm{~mm} / \mathrm{s}$. After the plotting speed was further increased, there were dots printed instead of continuous lines. Some of dots might be able to connect with adjacent ones, but the tracks are not interrupted. As plotting speed increased further, only silver dots were printed. Fig. 5 (c) showed discontinuous silver tracks and dots tracks with plotting speed increased from $13.5 \mathrm{~mm} / \mathrm{s}$ to 16.5 $\mathrm{mm} / \mathrm{s}$. The diameter of dots reflects the actual capability of printing system since they are formed by droplets.

The ratio of diameter of nozzle and diameter of falling droplets was about 7.6. During the printing process, the accumulated charges in the meniscus will result in formation of droplets. Assuming the current in meniscus is stable and proportion to voltage, the total amount of charges in a droplet can be estimated by integral of electrical voltage and time. It turned out to be greater line width with increased amplitude of voltage and decreased frequency (increased cycle time), which means more charges accumulate during the time interval. 


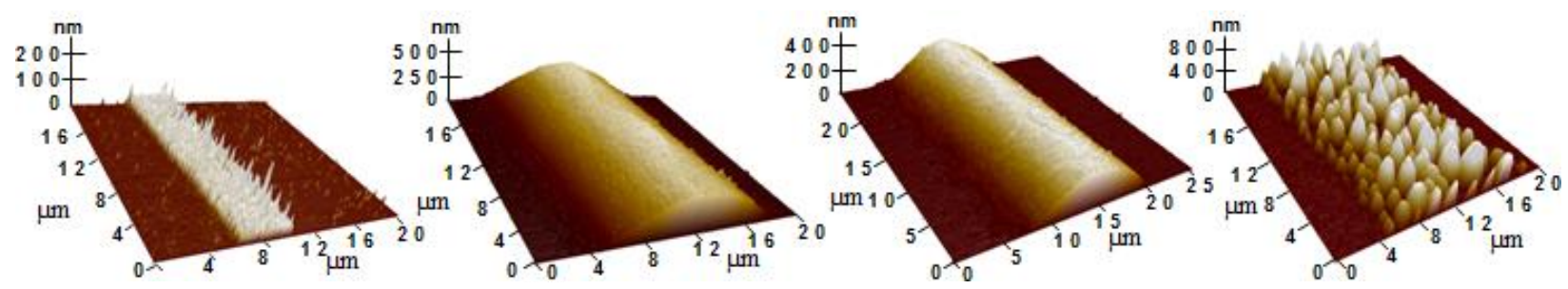

Fig. 6: (a) 3D AFM image of single layer pattern, (b) 3D AFM image of 20 layers pattern, (c) 3D AFM image of 20 layers pattern cured with ramped temperature to $220^{\circ} \mathrm{C}$, (d) $3 \mathrm{D}$ AFM image of 20 layers pattern cured instantly to $220^{\circ} \mathrm{C}$

\subsubsection{Conductivity characterization}

The silver ink consists of triethylene glycol, surfactants and silver nanoparticles. Nano particles are dispersed in the solvent, which would be evaporated during and soon after printing process. However, with smaller diameter of droplets to achieve better resolution, when they fall onto the substrate, they will splash out and transform into single particle bunches, as shown in Fig. 6 (a). Since most of bunches are not interconnected, there is no electric conductivity of single layer silver tracks. By depositing multi layers on the same position, we can print 20 layers silver tracks with fine semi-ellipse cross section, as shown in Fig. 6 (b). The cross sectional measured maximum height of $60 \mathrm{~nm}$ for the single layer pattern with an average thickness of $27 \mathrm{~nm}$ and line width of $5.3 \mu \mathrm{m}$. Because the geometric diameter of silver nanoparticles is below $50 \mathrm{~nm}$, it was believed that there was only single nano particle printed on the substrate without any overlay. That was the reason why the inter connectivity is so poor that single layer silver tracks could not carry a current flow. The cross sectional measured maximum height of $310 \mathrm{~nm}$ for the 20 layers pattern with an average thickness of $167 \mathrm{~nm}$ and line width of $15.1 \mu \mathrm{m}$. By depositing the silver ink on the same position to acquire multi-layer pattern, line width, line thickness and connectivity were increased with sacrifice of resolution. After curing process, the 20 layers silver track showed good electric conductivity.

To evaluate the effect of curing temperature on resistivity of micro silver tracks, a ramped curing temperature and instant curing temperature were first compared, as shown in Fig. 6 (c) and (d). Fig. 6 (c) was a 20 layers pattern which was gradually cured with ramped temperature from $40{ }^{\circ} \mathrm{C}$ to $220^{\circ} \mathrm{C}$ for 30 minutes and kept at $220^{\circ} \mathrm{C}$ for 15 minutes. The sintering process resulted in a fine recomposition of silver nanoparticles. However, if the temperature was instantly raised to $220{ }^{\circ} \mathrm{C}$ for 30 minutes, the high temperature caused drastic crystallization. Fig. 6 (d) demonstrated adjacent nanoparticles formed their own grains into larger particles instead of smoothly formed continuous silver tracks. These independent hillocks were not connected with each other, resulting in poor connectivity and insulation.

The curing process involves heat transport to create the necks between nanoparticles and transform them into grain boundaries. Diffusion is the principal mechanism. Lubricants and surfactant polymers are burned off first and nanoparticles are contacted with each other with a point bonding. As time goes on, necks are generated and then transformed into grain boundaries, a denser structure with a remarkable reduction in pores and interstices.

Shrinkage occurs during curing process as results of pore size reduction, lubricants and surfactants evaporation. This largely depends on the composition of silver ink, temperature and time. Silver tracks were printed with 10 lays and 20 lays using the same nozzle with glass tip outer diameter of $7 \mu \mathrm{m}$, using an AC voltage with $130 \mathrm{~Hz}$ frequency and $820 \mathrm{~V}$ amplitude. The silver tracks were cured under temperatures of $140{ }^{\circ} \mathrm{C}, 160{ }^{\circ} \mathrm{C}, 180^{\circ} \mathrm{C}, 200^{\circ} \mathrm{C}$ and $220^{\circ} \mathrm{C}$ using ramped pattern. There was a reduction in both line width and mean height (similar to maximum height) due to shrinkage. As maximum curing temperature went high, the shrinkage was more obvious. A maximal $14.2 \%$ reduction of line width and 
$17.8 \%$ reduction of mean height were observed in 10 layers sample. A maximal $10.2 \%$ reduction of line width and $14.5 \%$ reduction of mean height were observed in 20 layers sample.

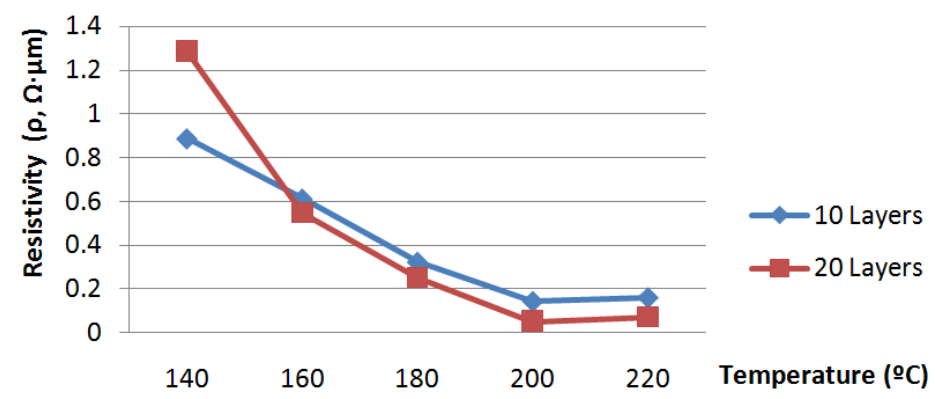

Fig. 7: Resistivity of different printed tracks with different curing temperatures

The resistance was measured with the help of an ohmmeter (Fluke 115) by contacting two probes on sintered silver tracks. Five measurements were taken at each sample at five different locations and then averaged. The electrical resistivity $\rho$ of the printed silver patterns was calculated by using the formula " $\rho$ $=R \times A / L$ ", where " $R$ " is the resistance of the pattern measured, " $A$ " is cross section area of the printed pattern and " $L$ " is the length between the two measuring points. The cross section $A$ of the silver tracks was calculated using equation " $A=w \times t$ ", where " $w$ " is the width of silver tracks and " $t$ " is the average thickness of the tracks, measured through AFM analysis. The electrical resistivity of printed patterns with different curing temperature is shown in Fig. 7. The electric resistivity of cured tracks decreases with increasing curing temperature and tended to be a stable value. The cured silver tracks have a minimum resistivity of $5.1 \mu \Omega \cdot \mathrm{cm}$ under a curing temperature at $200{ }^{\circ} \mathrm{C}$, which is about 3.16 times that of bulk silver. The result shows a good conductive property for silver patterns by e-jet printing. The resistivity was higher than bulk silver that is due to the calculation using the average thickness of the patterns and the development of void and cracks resulting from formation of coarse network.

\subsection{Electroless copper deposition on silver seeds}

Our plating bath contains two main chelating agents, TEA and EDTA, which are all able to chelate cupric copper ions. We investigated the effect of these two chelating agents. The initial concentration of dual-chelating agent will affect the growth rate and overall reaction of copper growth. The calibration of different variables and how they affect copper growth rate and cover rate were demonstrated in our previous research [41, 43]. Electroless copper deposition continuously consumes copper ions while chelating agents remain in the bath, which changes from their initial status.

We immersed cured silver patterns in solution with $24 \mathrm{~mL} / \mathrm{L}$ TEA, $12 \mathrm{~g} / \mathrm{L}$ EDTA for 30 minutes at $35^{\circ} \mathrm{C}$. The thickness of metal features was between $2 \mu \mathrm{m}$ and $15 \mu \mathrm{m}$, measured using profilometer. The thickness of the patterns was increased from original value around $250 \mathrm{~nm}$ of printed silver seed layer to 2-15 $\mu \mathrm{m}$ after copper deposition, about 10 times thinker. The average resistivity of copper lines was measured with the two point method to be $6.8 \mu \Omega \cdot \mathrm{cm}$, similar to printed silver patterns after curing process. The value is about four times bulk copper $(1.678 \mu \Omega \cdot \mathrm{cm})$ or bulk silver, which may come from the possible defects of the deposited copper layer. These defects in the copper structures could possibly come from the ambient laboratory conditions, where dust and other contaminants from atmospheric and plating solution can lead to defects. The edge resolutions of structures are determined by size of copper grains. There might be voids inside the metal structures that increase the resistivity.

For ultra large scale integration (ULSI), increasing current density is essential because improvement in interconnect time delay will offer significant improvements in the speed of the system. The time delay for an interconnect means the time for the voltage at one end of a metal line to come to $63 \%$ of its final value when an input is applied at the other end of the line. The delay time of an optimized interconnect line with length $L$ is proportional to its resistance $R$. As we mentioned in Section 3.1, the resistance of 
printed pattern can be calculated using formula " $R=\rho \times L / A$, and $A=w \times t$ ". For a pattern with length of $L$ and width $w$, the corresponding resistance $R$ will be reduced 10 times if thickness $t$ is increased 10 times. As a result, it has the ability to drive higher current and consume lower power.

Fig. 8 shows microscope pictures of microstructure of copper deposited on polyester (PET) film. Copper microstructures produced by combination of e-jet printing and electroless copper deposition on PET film were successfully fabricated. The average line width of printed features is around $20 \mu \mathrm{m}$ on PET film. Fig. 8 (a) shows microscopic pictures of part of silver patterns printed by e-jet printing with line width around $20 \mu \mathrm{m}$, followed by copper deposition as shown in Fig. 8 (b). It is clear from Figure 8(b) that copper is successfully grown on silver seeds.

As for the printed silver filaments without electroless copper deposition, these filaments have to be cured first in order to obtain high conductivity [32]. As we mentioned earlier, silver tracks were cured at $200^{\circ} \mathrm{C}$ for 20 minutes and then measured using two point method. However, as for flexible electronic fabrication techniques, $200{ }^{\circ} \mathrm{C}$ have already exceeded critical value of polymer-based substrates. That is a huge advantage if we can apply electroless copper deposition into the fabrication process to avoid curing of silver nanoparticles.

(a)

(b)

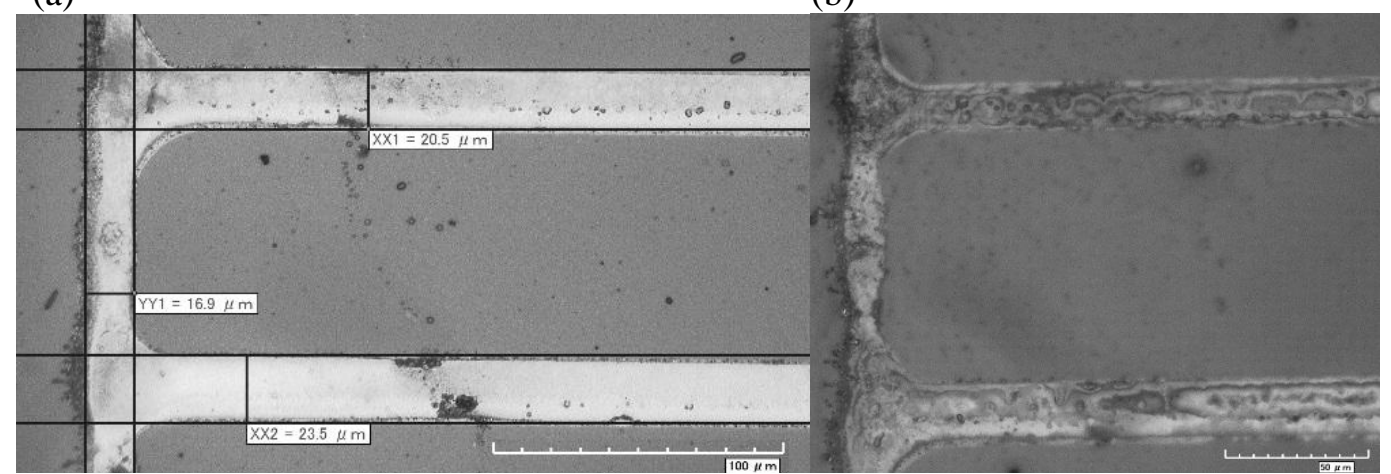

Fig. 8: High resolution conductive filaments matrix (a) printed by e-jet printing, and (b) after copper deposition. The thickness of printed patterns was increased from original around $250 \mathrm{~nm}$ silver seeds to 2$15 \mu \mathrm{m}$ with copper covered.

\subsection{Printing of electronic components}

In order to demonstrate the capability and versatility of our proposed e-jet printing followed by electroless copper deposition, we printed several electronic component patterns. As shown in Fig. 9 (a), using ac-pulse modulated e-jet printing, we successfully printed high resolution metal pads, interconnects for representative circuit patterns with critical dimensions as small as $18 \mu \mathrm{m}$ showed great potentials in microelectronic components fabrication. Fig. 9 (b) demonstrated silver tracks with line width of $12 \mu \mathrm{m}$ printed on the ABF. These can be applied for micro capacitor or inductor fabrication. The printed features are continuous, with micro-scale dimension, indicating charges accumulation problem are well solved using our technique.

Most lithograph methods are restricted to planar surfaces, which make it especially difficult to be extended to curved substrate [42]. The process presented in this paper doesn't have this limitation. We demonstrated the printing of silver seeds to generate patterns on curved surfaces and afterwards with copper deposition. The resolution of features on curved substrate is similar to that on planar substrates. Fig. 9 (c) shows microscope pictures of microstructure of copper generated on polyester (PET) film. Typical electrodes produced by combination of e-jet printing and electroless copper deposition on PET film were successfully fabricated. The average line width of features is $20 \mu \mathrm{m}$ with an average thickness of $7.3 \mu \mathrm{m}$ on PET film. 
(a)

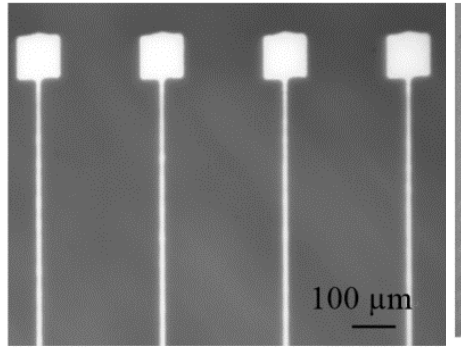

(b)

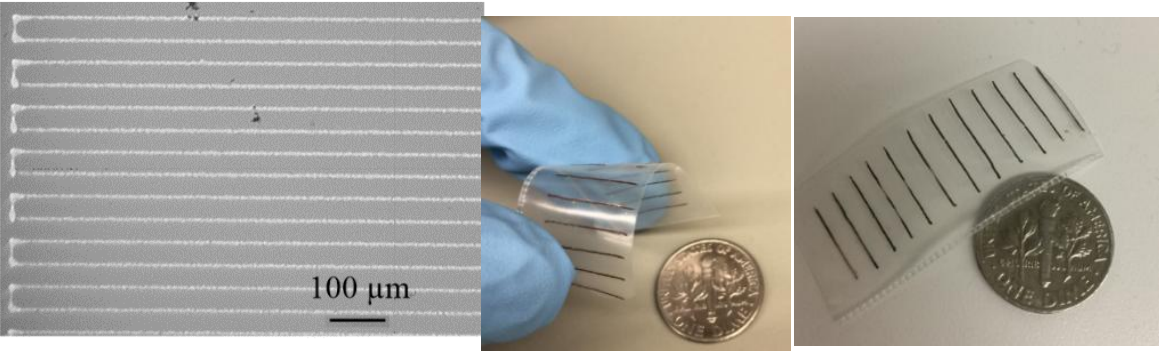

Fig. 9: Printed electronic components on highly insulating substrates: (a) printed metal pads and interconnects on $\mathrm{ABF}$ film, (b) an inductor pattern on $\mathrm{ABF}$ film, (c) Microstructures produced by combination of e-jet printing and electroless copper deposition on PET film with good conductivity; (d)

Printed conductive silver electrodes with copper shell compared to a Dime

\section{Conclusions}

In this paper, a fabrication method that integrates AC-pulse modulated e-jet printing and electroless copper deposition was presented for direct fabrication of highly conductive micro-scale patterns with sub$20 \mu \mathrm{m}$ resolution on highly insulating substrates and flexible substrates. The fabrication process and detailed mechanism of e-jet printing were discussed in the paper. Amplitude and frequency of AC-pulsed voltage, plotting speed, and electrical characterization were conducted in this research to minimize effect of residue charge, ensuring high-resolution printing of continuous patterns on insulating substrates. The minimum resistivity of printed track is $5.1 \mu \Omega \cdot \mathrm{cm}$, which is about 3.16 times that of bulk silver $(1.6 \mu$ $\Omega \cdot \mathrm{cm}$ ). By introducing electroless copper deposition, we successfully fabricated conductive patterns on flexible substrates with large pattern thickness without using high temperature curing process. Copper deposition were successfully applied on printed silver seeds to increase thickness of patterns up to $15 \mu \mathrm{m}$ with resistivity about four times bulk copper on flexible substrates. With the help of copper deposition to increase cross section area, the resistance of printed patterns can be significantly decreased. As a result, the patterns after deposition are capable to drive higher current and consume lower power. Thickness issues for e-jet printing are successfully resolved in the proposed process. The technique can be used on different substrates such as polymers and glass, both planar and curved surfaces, showing great potential in flexible electronics and biomedical applications. The presented technique is capable to fabricate resistors, inductors and micro wires, which offer a simple and versatile method to on demand direct fabricate conductive patterns in micro/nano electronic manufacturing. Results in this study provide a valuable method for electroless copper deposition for specific applications in flexible electronics.

\section{Acknowledgements}

This work was partially supported by the National Science Foundation (NSF) Grants (CMMI1125872, CMMI-1333775, CBET-1344618, and CMMI-1404916) to North Carolina State University. Their support is greatly appreciated. 


\section{References}

[1] W.S. Wong, A. Salleo, Flexible electronics: materials and applications, Springer, 2009.

[2] R. Parashkov, E. Becker, T. Riedl, H.-H. Johannes, W. Kowalsky, Large area electronics using printing methods, Proceedings of the IEEE, 93 (2005) 1321-1329.

[3] S.E. Burns, P. Cain, J. Mills, J. Wang, H. Sirringhaus, Inkjet printing of polymer thin-film transistor circuits, MRS bulletin, 28 (2003) 829-834.

[4] T. Shimoda, Y. Matsuki, M. Furusawa, T. Aoki, I. Yudasaka, H. Tanaka, H. Iwasawa, D. Wang, M. Miyasaka, Y. Takeuchi, Solution-processed silicon films and transistors, Nature, 440 (2006) 783-786.

[5] H. Sirringhaus, T. Kawase, R. Friend, T. Shimoda, M. Inbasekaran, W. Wu, E. Woo, High-resolution inkjet printing of all-polymer transistor circuits, Science, 290 (2000) 2123-2126.

[6] M.J. Heller, DNA microarray technology: devices, systems, and applications, Annual review of biomedical engineering, 4 (2002) 129-153.

[7] A. Bietsch, J. Zhang, M. Hegner, H.P. Lang, C. Gerber, Rapid functionalization of cantilever array sensors by inkjet printing, Nanotechnology, 15 (2004) 873.

[8] Z. Wang, H. Shang, G.U. Lee, Nanoliter-scale reactor arrays for biochemical sensing, Langmuir, 22 (2006) 6723-6726.

[9] J.-U. Park, M. Hardy, S.J. Kang, K. Barton, K. Adair, D. kishore Mukhopadhyay, C.Y. Lee, M.S. Strano, A.G. Alleyne, J.G. Georgiadis, High-resolution electrohydrodynamic jet printing, Nature materials, 6 (2007) 782-789.

[10] N. Stutzmann, R.H. Friend, H. Sirringhaus, Self-aligned, vertical-channel, polymer field-effect transistors, Science, 299 (2003) 1881-1884.

[11] J. Zeleny, Instability of electrified liquid surfaces, Physical Review, 10 (1917) 1.

[12] M. Cloupeau, B. Prunet-Foch, Electrohydrodynamic spraying functioning modes: a critical review, Journal of Aerosol Science, 25 (1994) 1021-1036.

[13] D.-Y. Lee, Y.-S. Shin, S.-E. Park, T.-U. Yu, J. Hwang, Electrohydrodynamic printing of silver nanoparticles by using a focused nanocolloid jet, Applied Physics Letters, 90 (2007) 081905-081905081903.

[14] D. Kim, S. Jeong, J. Moon, K. Kang, Ink-jet printing of silver conductive tracks on flexible substrates, Molecular Crystals and Liquid Crystals, 459 (2006) 45/[325]-355/[335].

[15] S.K. Volkman, Y. Pei, D. Redinger, S. Yin, V. Subramanian, Ink-jetted silver/copper conductors for printed RFID applications, in: MRS Proceedings, Cambridge Univ Press, 2004, pp. I7. 8.

[16] S. Lee, J. Song, H. Kim, J. Chung, Time resolved imaging of electrohydrodynamic jetting on demand induced by square pulse voltage, Journal of Aerosol Science, (2012).

[17] J.H. Yu, S.Y. Kim, J. Hwang, Effect of viscosity of silver nanoparticle suspension on conductive line patterned by electrohydrodynamic jet printing, Applied Physics A, 89 (2007) 157-159.

[18] S.-H. Lee, X.H. Nguyen, H.S. Ko, Study on droplet formation with surface tension for electrohydrodynamic inkjet nozzle, Journal of mechanical science and technology, 26 (2012) 1403-1408. [19] V.D. Nguyen, D. Byun, Mechanism of electrohydrodynamic printing based on ac voltage without a nozzle electrode, Applied Physics Letters, 94 (2009) 173509.

[20] C.-C. Yang, C.-C. Wan, Y.-Y. Wang, Synthesis of Ag/Pd nanoparticles via reactive micelles as templates and its application to electroless copper deposition, Journal of colloid and interface science, 279 (2004) 433-439.

[21] N. Fritz, H.-C. Koo, Z. Wilson, E. Uzunlar, Z. Wen, X. Yeow, S.A.B. Allen, P.A. Kohl, Electroless deposition of copper on organic and inorganic substrates using a Sn/Ag catalyst, Journal of The Electrochemical Society, 159 (2012) D386-D392.

[22] P.C. Hidber, W. Helbig, E. Kim, G.M. Whitesides, Microcontact printing of palladium colloids: micron-scale patterning by electroless deposition of copper, Langmuir, 12 (1996) 1375-1380. 
[23] A. Khan, K. Rahman, M.-T. Hyun, D.-S. Kim, K.-H. Choi, Multi-nozzle electrohydrodynamic inkjet printing of silver colloidal solution for the fabrication of electrically functional microstructures, Applied Physics A, 104 (2011) 1113-1120.

[24] D.-Y. Lee, J.-C. Lee, Y.-S. Shin, S.-E. Park, T.-U. Yu, Y.-J. Kim, J. Hwang, Structuring of conductive silver line by electrohydrodynamic jet printing and its electrical characterization, in: Journal of physics: conference series, IOP Publishing, 2008, pp. 012039.

[25] D.-Y. Lee, D.-H. Lim, Y.-J. Kim, J. Hwang, Electrical Characterization of a Microstrip Line Patterned by Electrohydrodynamic Jet Printing of Silver Nanoparticles, Journal of Imaging Science, 53 (2009) 41203-41203.

[26] K. Rahman, A. Khan, N.M. Muhammad, J. Jo, K.-H. Choi, Fine-resolution patterning of copper nanoparticles through electrohydrodynamic jet printing, Journal of Micromechanics and Microengineering, 22 (2012) 065012.

[27] M.W. Lee, N.Y. Kim, S. An, J.-Y. Huh, H.Y. Kim, S.S. Yoon, Effects of pulsing frequency on characteristics of electrohydrodynamic inkjet using micro-Al and nano-Ag particles, Experimental Thermal and Fluid Science, (2012).

[28] P. Galliker, J. Schneider, H. Eghlidi, S. Kress, V. Sandoghdar, D. Poulikakos, Direct printing of nanostructures by electrostatic autofocussing of ink nanodroplets, Nature communications, 3 (2012) 890.

[29] B. Kim, H. Nam, S.J. Kim, J. Sung, S.W. Joo, G. Lim, Control of charged droplets using electrohydrodynamic repulsion for circular droplet patterning, Journal of Micromechanics and Microengineering, 21 (2011) 075020.

[30] M. Lee, D. Kang, N. Kim, H. Kim, S. James, S. Yoon, A study of ejection modes for pulsed-DC electrohydrodynamic inkjet printing, Journal of Aerosol Science, 46 (2012) 1-6.

[31] H.T. Yudistira, V.D. Nguyen, P. Dutta, D. Byun, Flight behavior of charged droplets in electrohydrodynamic inkjet printing, Applied Physics Letters, 96 (2010) 023503.

[32] H. Qin, C. Wei, J. Dong, Y.-S. Lee, Direct Fabrication of Highly Conductive Micro Silver Tracks Using Electrohydrodynamic Jet Printing for Sub-20 $\mu$ M Micro-Manufacturing, in: Proceedings of 2014 ASME International Manufacturing Science and Engineering Conference, 2014, Paper Number 4163. [33] C. Wei, H. Qin, N.A. Ramírez-Iglesias, C.-P. Chiu, Y.-s. Lee, J. Dong, High-resolution ac-pulse modulated electrohydrodynamic jet printing on highly insulating substrates, Journal of Micromechanics and Microengineering, 24 (2014) 045010.

[34] C. Wei, H. Qin, C.-P. Chiu, Y.-S. Lee, J. Dong, Modulated Drop-on-Demand E-jet Printing of Continuous Features on Electronic Insulation Substrates, in: Proceeding of NAMRI/SME North American Manufacturing Research Conference, 2014, (CD) Paper Number 4476.

[35] C. Wei, H. Qin, C.-P. Chiu, Y.-S. Lee, J. Dong, Drop-on-demand E-jet printing of continuous interconnects with AC-pulse modulation on highly insulating substrates, accepted for publication in Journal of Manufacturing Systems, (2014), in press.

[36] M. Schlesinger, M. Paunovic, Modern electroplating, John Wiley \& Sons, 2011.

[37] G.O. Mallory, J.B. Hajdu, Electroless plating: fundamentals and applications, William Andrew, 1990.

[38] Y. Shacham-Diamand, V. Dubin, M. Angyal, Electroless copper deposition for ULSI, Thin Solid Films, 262 (1995) 93-103.

[39] Y.-M. Lin, S.-C. Yen, Effects of additives and chelating agents on electroless copper plating, Applied Surface Science, 178 (2001) 116-126.

[40] Z. Hu, T. Ritzdorf, Electroless Deposition Processes and Tools, in: Advanced Nanoscale ULSI Interconnects: Fundamentals and Applications, Springer, 2009, pp. 413-433.

[41] H. Qin, J. Dong, Y.-S. Lee, Electroydrodynamic Jet Printing of Silver Seeds: Micro Scale Patterning

by Electrolyses Copper Deposition, in: Accepted to appear in Proceedings of the ASME 2015

Manufacturing Science and Engineering Conference, Charlotte, North Carolina, 2015, (CD) Paper Number 9487.

[42] H.-H. Hsu, K.-H. Lin, S.-J. Lin, J.-W. Yeh, Electroless copper deposition for ultralarge-scale integration, Journal of The Electrochemical Society, 148 (2001) C47-C53. 
[43] C. Wei, J. Dong, "Development and Modeling of Melt Electrohydrodynamic-Jet Printing of PhaseChange Inks for High-Resolution Additive Manufacturing”, ASME Journal of Manufacturing Science and Engineering, 136(6), 061010, 2014 


\section{Glossary}

\begin{tabular}{|c|c|}
\hline$d$ & line width of printed tracks, diameter of printed droplets \\
\hline$d_{0}$ & actual diameter of falling droplets \\
\hline$V_{1}$ & volume of printed droplets \\
\hline$V_{0}$ & actual volume of falling droplets \\
\hline$h$ & average height of printed droplets \\
\hline$f$ & frequency of $\mathrm{AC}$ voltage \\
\hline$T$ & circle time of AC voltage \\
\hline$T_{d}$ & time between two adjacent droplets \\
\hline$v_{\max }$ & maximum plotting speed for continuous silver tracks \\
\hline$\rho$ & electrical resistivity of printed silver patterns \\
\hline$R$ & resistance of pattern measured \\
\hline$A$ & cross section area of printed patterns \\
\hline$L$ & length between two measuring points \\
\hline$w$ & width of silver tracks \\
\hline
\end{tabular}

